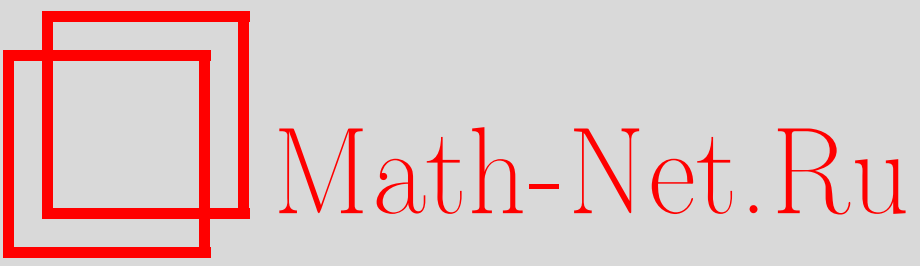

В. В. Зверев, Об условиях существования интегралов по фрактальным носителям, ТМФ, 1996, том 107, номер 1, 3-11

DOI: https://doi.org/10.4213/tmf1133

Использование Общероссийского математического портала Math-Net.Ru подразумевает, что вы прочитали и согласны с пользовательским соглашением

http://www.mathnet.ru/rus/agreement

Параметры загрузки:

IP: 54.80 .97 .219

26 апреля 2023 г., 02:45:57

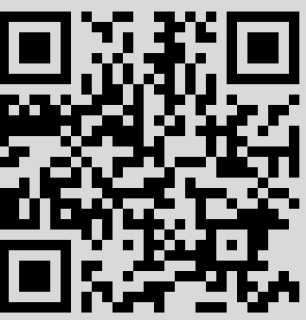


ТЕОРЕТИЧЕСКАЯ

И МАТЕМАТИЧЕСКАЯ

ФИЗИКА

Том 107, № 1

апрель, 1996

В.В. Зверев

\section{ОБ УСЛОВИЯХ СУЩЕСТВОВАНИЯ ИНТЕГРАЛОВ ПО ФРАКТАЛЬНЫМ НОСИТЕЛЯМ}

Показано, что процессы преобразования шума в некоторых системах с задержкой могут быть описаны посредством двух типов интегралов по фрактальным носителям. Проанализированы условия сходимости таких интегралов. Рассмотрена структура мультифракталов, ассоциированных с интегралами, при различных коэффициентах сжатия.

\section{1.ВВЕДЕНИЕ}

Широкие исследования самоподобных структур (“физических” фракталов), нашедшие отражение в многочисленных публикациях последних лет (недавние обзоры на эту тему - $[1,2]$ ), были в определенной степени стимулированы рядом предшествуюших теоретических работ, разъяснявших и популяризовавших идеи и методы фрактальной геометрии (отметим пионерские работы Мандельброта [3, 4], а также работы [5-8]). Одна из главных идей состоит в соотнесении натурного объекта с нестандартным множеством, родственным множеству Кантора и имеющим дробную размерность ("математическим" фракталом), либо с сингулярным распределением некоторой величины на таком множестве (т.е. с мультифрактальной мерой, мультифракталом [9]). Таким образом, канторовский фрактал приобретает статус идеализированной модели реальной среды, а средние по фракталу играют роль физических величин. Описанная схема теоретического анализа, не вызывая каких-либо обших возражений, должна тем не менее использоваться с известной осторожностью. Следует учитывать, что в определенной ситуации продление иерархии уровней самоподобия до бесконечности может приводить к появлению проблем со сходимостью средних. Выполнение расчетов на "физическом" уровне строгости чревато в этом случае получением ошибочных результатов. В данной работе мы продемонстрируем это, рассматривая простой тип интеграла по мультифракталу $(И М \Phi)$ и выясняя условия его сходимости.

Определим ИМФ от $f(x), x \in[0,1]$, выражением

$$
\int_{\mathcal{L}} f(x) d \mu(x \mid \kappa, \Theta)=\lim _{n \rightarrow \infty} \sigma_{n}=\lim _{n \rightarrow \infty} \sigma_{n}^{\#},
$$

где

$$
\begin{aligned}
\sigma_{n} & =2^{-n} \sum_{s} \Theta_{n}^{[s]} f\left(\lambda_{n}^{[s]}\right), \\
\sigma_{n}^{\#} & =2^{-n} \sum_{s} \Theta_{n}^{[s]}\left\langle f\left(\lambda_{n}^{[s]}\right)\right\rangle_{\kappa^{n}}
\end{aligned}
$$


(условия, при которых пределы в (1) сушествуют и равны, будут установлены в разделе 3). Здесь $\mathbf{s}_{n}=\left(s_{n}^{(1)}, \ldots, s_{n}^{(n)}\right)$ - вектор сигнатуры, имеюший компоненты $s_{n}^{(i)}= \pm 1$; суммирование в (2), (3) ведется по множеству таких векторов. Аргумент функции принимает значения

$$
\lambda_{n}^{[s]}=\frac{1}{2}+\frac{1}{2}(1-\kappa)\left(\mathbf{s}_{n}, \mathbf{h}_{n}(\kappa)\right)
$$

где $\mathbf{h}_{n}=\left(1, \kappa, \kappa^{2}, \ldots, \kappa^{n-1}\right), \kappa<1$; символом $(\mathbf{a}, \mathbf{b})$ обозначено скалярное произведение. Усреднение в (3) производится по формуле

$$
\langle f(x)\rangle_{\delta}=\frac{1}{\delta} \int_{x-\delta / 2}^{x+\delta / 2} f(t) d t .
$$

Величины $\Theta_{n}^{[s]}$ являются функциями от $s$. Далее мы будем считать, что имеет место условие нормировки: $\sigma_{n}=\sigma_{n}^{\#}=1$ при $f \equiv 1$. В этом случае должно выполняться равенство

$$
\Theta_{n}^{\left[s^{\prime}\right]}+\Theta_{n}^{\left[s^{\prime \prime}\right]}=2 \Theta_{n-1}^{[s]}
$$

для $\mathbf{s}_{n}^{\prime}=\mathbf{s}_{n-1} \oplus(+1), \mathbf{s}_{n}^{\prime \prime}=\mathbf{s}_{n-1} \oplus(-1)$ (операция $\oplus$ состоит в “срашивании" векторов). Условимся называть (1) ИМ $\Phi$ 1-го рода, если выполнены условия

$$
\begin{array}{ll}
\Theta_{n}^{[s]}=\prod_{i=1}^{n} \psi\left(s_{n}^{(i)}\right), & \psi(+1)+\psi(-1)=2, \\
\operatorname{Im} \psi( \pm 1)=0, & \operatorname{Re} \psi( \pm 1)>0,
\end{array}
$$

и ИМФ 2-го рода, если выполнены условия (7a), а хотя бы одно из условий (7б) нарушено. В первом случае величины $p_{ \pm}=\frac{1}{2} \psi( \pm 1)$ могут интерпретироваться как вероятности; соответствующие мультифрактальные меры (порождаемые мультипликативным процессом Безиковича) рассматривались в [9].

Ниже в разделе 2 мы покажем, что ИМФ как 1-го, так и 2-го рода возникают при решении задачи о стационарном шуме в системах с запаздыванием. В разделе 3 найдены условия сходимости интегралов, а в разделе 4 рассмотрена структура носителя при различных $\kappa$.

\section{2. ПРЕОБРАЗОВАНИЕ ШУМА В СИСТЕМАХ С ЗАПАЗДЫВАНИЕМ}

2.1. Линейная система с дихотомическим и гауссовским шумом. Будем считать, что преобразование двумерного (комплексного) шума в линейной системе с запаздыванием описывается разностным уравнением

$$
X(t)=\kappa X(t-\tau)+\xi(t-\tau)
$$

при $\kappa \in \mathbb{R}, \kappa<1$, причем $\xi=\xi_{\mathrm{g}}+\xi_{d}$, где $\xi_{\mathrm{g}}-$ гауссовский случайный процесс $(\mathrm{C \Pi})$ и $\xi_{\mathrm{d}}-$ дихотомический СП (частный случай СП Кубо-Андерсена $[10,11])$. При выполнении 
условия для времени корреляции шума $\tau_{\text {сог }} \ll \tau$ функция распределения переменной $X$ удовлетворяет уравнению типа Колмогорова-Чепмена [12]

$$
P_{N+1}(X)=\int d Y\left\{P_{\mathrm{g}} * P_{\mathrm{d}}\right\}(X-\kappa Y) P_{N}(Y),
$$

где

$$
\begin{gathered}
P_{\mathrm{g}}(X, R)=(\pi R)^{-1} e^{-|x|^{2} / R} ; \quad P_{\mathrm{d}}(X)=\sum_{i=1}^{2} p_{i} \delta^{(2)}\left(X-A_{i}\right) \\
P_{N}(X) \equiv P\left(t_{0}+N \tau, X\right), \quad t_{0} \in[0, \tau), \quad N \in \mathbb{N} ; \\
\delta^{(2)}(X)=\delta(\operatorname{Re} X) \delta(\operatorname{Im} X) ; \quad R=\left\langle\left|\xi_{\mathrm{g}}\right|^{2}\right\rangle
\end{gathered}
$$

символом * обозначена свертка. Переходя к уравнению для характеристических функций и решая его методом итераций, нетрудно представить стационарное решение в виде

$$
P_{\mathrm{st}}(X)=\lim _{N \rightarrow \infty} P_{N}(X)=\int_{\mathcal{L}} P_{\mathrm{g}}\left(X-\frac{A_{1} x+A_{2}(1-x)}{1-\kappa}, \frac{R}{1-\kappa^{2}}\right) d \mu(x \mid \kappa, \Theta) .
$$

Полученное выражение содержит ИМФ 1-го рода $\left(\frac{1}{2} \psi(+1)=p_{1}, \frac{1}{2} \psi(-1)=p_{2}\right)$.

2.2. Система с нелинейным преобразованием Икеды и гауссовским шумом. Рассмотрим нелинейное преобразование гауссовского шума, которое определяется уравнением

$$
X(t)=F(X(t-\tau))+\xi_{\mathrm{g}}(t-\tau),
$$

где $F(X)=\kappa X \exp \left(i \lambda|X|^{2}+i \phi_{0}\right)+1$ (преобразование Икеды $\left.[13,14]\right)$. При $\tau_{\text {сог }} \ll \tau$ уравнение для функций распределения имеет вид

$$
P_{N+1}(X)=\iint d Y d Z P_{\mathrm{g}}(X-Y) \delta^{(2)}(Y-F(Z)) P_{N}(Z) .
$$

Как было показано в работе [12], в пределе сильного перемешивания $\lambda \gg 1$ можно воспользоваться приближением случайных фаз, заменяя в (12)

$$
\delta^{(2)}(Y-F(Z)) \longrightarrow\left\langle\delta^{(2)}\left(Y-1-\kappa Z e^{i \phi}\right)\right\rangle
$$

где $\langle\ldots\rangle$ - усреднение по $\phi$. В этом случае

$$
P_{\mathrm{st}}(X)=\int d Y P_{\mathrm{g}}\left(X-Y, \frac{R}{1-\kappa^{2}}\right) P_{\mathrm{ch}}(Y) .
$$

Радиально-симметричное распределение $P_{\mathrm{ch}}$, описьваюшее шум, обусловленный динамической хаотизацией движения [12], может быть записано в виде

$$
P_{\mathrm{ch}}(X)=\left(\pi \kappa^{2}\right)^{-1} f_{\mathrm{ch}}\left(|X-1|^{2} \kappa^{-2}\right),
$$

Где

$$
f_{\mathrm{ch}}(Q)=\frac{1}{2} \int_{0}^{\infty} \beta d \beta J_{0}\left(\beta Q^{1 / 2}\right) \prod_{\gamma=0}^{\infty} J_{0}\left(\beta \kappa^{\gamma}\right)
$$




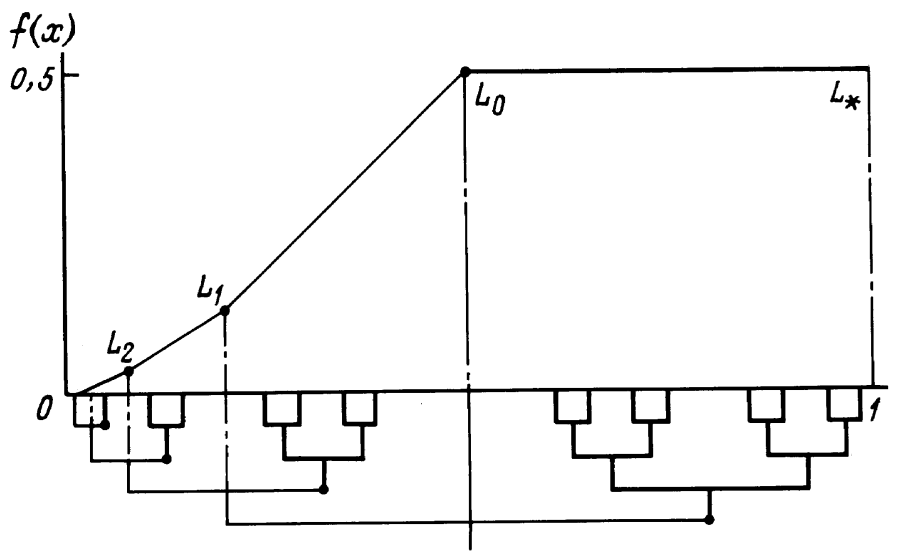

Изображение графа, отражающего процесс формирования предфрактала порядка $n=4$ при $\kappa=0,4$, и графика функции, определенной в пункте 3.3 . Вклады линейных участков графика получаются в результате суммирования по вершинам кластеров, выделенных жирными линиями

С целью дальнейшего упрошения выражения (16) введем приближение, заменяя функцию Бесселя наиболее близкой функцией с разделенными “колебательной” и монотонной частями: $J_{0}(x) \approx \chi(x)\left[\sqrt{2} \cos \left(x-\frac{1}{4} \pi\right)\right]$. В этом приближении

$$
f_{\mathrm{ch}}(Q) \approx \frac{1}{\sqrt{2}}\left\{e^{-i \pi / 4} \int_{\mathcal{L}} \hat{\Phi}\left(\sqrt{Q}+\frac{2 x-1}{1-\kappa}, Q\right) d \mu(x \mid \kappa, \Theta)+\text { к.c. }\right\}
$$

где $\hat{\Phi}$ - фурье-образ (по первому аргументу) монотонной функции

$$
\Phi(\beta, Q)=\frac{1}{2} \beta \chi(\beta \sqrt{Q}) \prod_{\gamma=0}^{\infty} \chi\left(\beta \kappa^{\gamma}\right)
$$

$$
\Theta_{n}^{[s]}=2^{n / 2} \exp \left[-\frac{i \pi}{4}\left(\mathbf{s}_{n}, \mathbf{h}_{n}(1)\right)\right]
$$

В (17) входит ИМФ 2-го рода, т.к. величины (19) не удовлетворяют условию (7б).

\section{3. УСЛОВИЯ СХОДИМОСТИ ИМФ}

При фиксированном $n$ суммирование в (2) проводится по всем точкам предфрактала $n$-го поколения, роль которого в данном случае играет множество точек, имеющих координаты $\lambda_{n}^{[s]} \in(0,1)$; число таких точек равно числу различных $\mathbf{s}_{n}$, т.е. $2^{n}$. Располагая предфракталы друг под другом в порядке возрастания $n$ и соединяя точки по правилу $\mathbf{s}_{n} \rightarrow \mathbf{s}_{n+1}^{ \pm}=\mathbf{s}_{n} \oplus( \pm 1)$, получаем граф, изображающий процесс формирования канторовского множества, возникающего в пределе $n \rightarrow \infty$ (см. рисунок). Заметим, что если граф̆ включает в себя предфракталы первых $n+k$ поколений, каждая точка предфрактала $n$-го поколения является вершиной-предком для кластера, включаюшего 
в себя точки последующих поколений. При $k \rightarrow \infty$ каждый такой кластер сходится к "малому" канторовскому фракталу, который можно совместить с полным предельным множеством, выполняя соответствующие растяжение и смешение. Выделяя в суммировании по $n+k$-му предфракталу вклады кластеров, можно записать

$$
\left|\sigma_{n+k}-\sigma_{n+k^{\prime}}\right|=\left|\omega_{n, k}-\omega_{n, k^{\prime}}\right|
$$

где

$$
\omega_{n, k}=2^{-n} \sum_{s}\left(2^{-k} \sum_{r} \Theta_{n+k}^{[s \oplus r]}\left[f\left(\lambda_{n}^{[s]}+\Delta_{n, k}^{[r]}\right)-f\left(\lambda_{n}^{[s]}\right)\right]\right)
$$

и $\Delta_{n, k}^{[r]}=\frac{1}{2} \kappa^{n}(1-\kappa)\left(\mathbf{r}_{k}, \mathbf{h}_{k}(\kappa)\right)$ (члены с минусом в квадратных скобках добавлены искусственно). Далее доказательство сходимости будет состоять в выполнении оценок для (20) при большом $n$ и использовании критерия Коши.

3.1. Случай ИМФ 1-го рода. Если выполнены условия (7), то $\left|\Theta_{n}^{[s]}\right| \equiv \Theta_{n}^{[s]}>0$ и $\left|\sigma_{n+k}-\sigma_{n+k^{\prime}}\right| \leq \Omega_{n, k}+\Omega_{n, k^{\prime}}$, где

$$
\Omega_{n, k}=2^{-n} \sum_{s}\left(2^{-k} \sum_{r} \Theta_{n+k}^{[s \oplus r]}\left|f\left(\lambda_{n}^{[s]}+\Delta_{n, k}^{[r]}\right)-f\left(\lambda_{n}^{[s]}\right)\right|\right) .
$$

Предположим, что функция $f(x)$ непрерывна на каждом предфрактале:

$$
(\forall n)\left(\forall \mathbf{s}_{n}\right)(\forall \varepsilon)\left(\exists \delta_{\varepsilon, n}^{[s]}\right)\left(\forall \delta<\delta_{\varepsilon, n}^{[s]}\right):\left|f\left(\lambda_{n}^{[s]}+\delta\right)-f\left(\lambda_{n}^{[s]}\right)\right|<\varepsilon / 2 .
$$

Учитывая, что $\left|\Delta_{n, k}^{[r]}\right|<(1 / 2) \kappa^{n}$ и (поскольку число точек каждого предфрактала конечно) $\exists \delta_{\varepsilon, n}=\min _{s} \delta_{\varepsilon, n}^{[s]}$, сформулируем условие для $\delta_{\varepsilon, n}$ : если $\left(\exists n_{\varepsilon}\right)\left(\forall n>n_{\varepsilon}\right)$ $\left(\delta_{\varepsilon, n}>(1 / 2) \kappa^{n}\right)$, то

$$
(\forall \varepsilon)\left(\exists n_{\varepsilon}\right)\left(\forall n>n_{\varepsilon}\right):\left|\sigma_{n+k}-\sigma_{n+k^{\prime}}\right|<\varepsilon,
$$

т.е. последовательность $\left\{\sigma_{n}\right\}$ является фундаментальной и сходящейся. В частности, если $f(x)$ - непрерывная на $[0,1]$ (и, следовательно, равномерно непрерывная) функция, $\exists \delta_{\varepsilon}=\min _{n} \delta_{\varepsilon, n}$ и $(24)$ также выполняется.

Применяя к интегралу (5) теорему о среднем, можно получить

$$
\left|\sigma_{n}-\sigma_{n}^{\#}\right| \leq 2^{-n} \sum_{s} \Theta_{n}^{[s]}\left|f\left(\lambda_{n}^{[s]}+\tilde{\Delta}_{n}^{[s]}\right)-f\left(\lambda_{n}^{[s]}\right)\right|,
$$

где $\left|\tilde{\Delta}_{n}^{[s]}\right| \leq(1 / 2) \kappa^{n}$. Далее по аналогии с вышеизложенным, нетрудно показать, что при тех же условиях, наложенных на функции, имеет место равенство пределов (1).

Таким образом, справедливо

ПРЕДЛОЖЕНИЕ 1. Если функиия $f(x), x \in[0,1]$, непрерывна и выполнены условия (7a), (7б), последовательности величин (2), (3) сходятся к общему пределу, являющемуся ИМФ 1-го рода.

3.2. Случай ИМФ 2-го рода. Предположим теперь, что выполнено условие (7a), но $\psi(+1)$ - произвольное комплексное число. Обозначим

$$
\sigma_{n}^{(t)}=2^{-n} \sum_{s} \Theta_{n}^{[s]} f^{(t)}\left(\lambda_{n}^{[s]}\right)
$$


где $f^{(t)}(x)$ - производная функции порядка $t$, и покажем, что последовательность величин (26) ограничена, если $f(x)$ дифференцируема бесконечное число раз и $(\forall t \in \mathbb{N})$ : $\left|f^{(t)}(1 / 2)\right|<M$. Разлагая $f^{(t)}(x)$ в степенной ряд около точки $1 / 2$ и учитывая (4), получим

$$
\left|\sigma_{n}^{(t)}\right| \leq \sum_{j=0}^{\infty} \frac{1}{j !}\left|f^{(t+j)}\left(\frac{1}{2}\right)\right|\left(\frac{1-\kappa}{2}\right)^{j}\left|2^{-n} \sum_{s} \Theta_{n}^{[s]}\left(\mathbf{s}_{n}, \mathbf{h}_{n}(\kappa)\right)^{j}\right| .
$$

Вводя производящую функцию для степеней скалярного произведения $\exp \left[\zeta\left(\mathbf{s}_{n}, \mathbf{h}_{n}(\kappa)\right)\right]$, преобразуем последний сомножитель в (27), заменяя сумму по $\mathbf{s}_{n}$ произведением двучленов. В итоге будем иметь

$$
2^{-n} \sum_{s} \Theta_{n}^{[s]}\left(\mathbf{s}_{n}, \mathbf{h}_{n}(\kappa)\right)^{j}=\left.\frac{d^{j}}{d \zeta^{j}} \prod_{i=1}^{n}\left(\frac{1}{2} \sum_{p= \pm 1} \psi(p) e^{p \zeta \kappa^{i-1}}\right)\right|_{\zeta=0} .
$$

Используя формулу Лейбница, получим из (28) оценку

$$
\left|2^{-n} \sum_{s} \Theta_{n}^{[s]}\left(\mathbf{s}_{n}, \mathbf{h}_{n}(\kappa)\right)^{j}\right| \leq \sum_{\mathbf{m}} C_{j}^{\mathbf{m}} \prod_{i=1}^{n}\left|\frac{1}{2} \sum_{p= \pm 1} p^{m_{i}} \psi(p)\right| \kappa^{(i-1) m_{i}},
$$

здесь $C_{j}^{\mathbf{m}}=j ! / \prod_{i=1}^{n} m_{i}$ ! и сумма берется по целым неотрицательным $m_{i}$, удовлетворяющим условию $\sum_{i=1}^{n} m_{i}=j$. Заметим, что число сомножителей $(1 / 2)[\psi(+1)-\psi(-1)]$ в каждом из произведений, суммируемых в правой части (29), не превышает наименьшего из чисел $n, j$. Пользуясь этим и обозначая $G=\max \{(1 / 2)[\psi(+1)-\psi(-1)], 1\}$, получим из (29)

$$
\left|2^{-n} \sum_{s} \Theta_{n}^{[s]}\left(\mathbf{s}_{n}, \mathbf{h}_{n}(\kappa)\right)^{j}\right| \leq G^{j} \sum_{\mathbf{m}} C_{j}^{\mathbf{m}} \prod_{i=1}^{n} \kappa^{(i-1) m_{i}} \leq G^{j}(1-\kappa)^{-j} .
$$

Теперь, используя (30) и учитывая ограниченность производных, найдем из (27)

$$
\left|\sigma_{n}^{(t)}\right| \leq M e^{G / 2}
$$

Чтобы получить оценку для (20), воспользуемся представлением (20), (21), подставляя туда $f(x)$ в виде степенного разложения. В результате будем иметь $\mid \sigma_{n+k}-$ $\sigma_{n+k^{\prime}} \mid \leq \tilde{\Omega}_{n, k}+\tilde{\Omega}_{n, k^{\prime}}$, где

$$
\tilde{\Omega}_{n, k}=\sum_{j=1}^{\infty} \frac{1}{j !}\left[\frac{\kappa^{n}(1-\kappa)}{2}\right]^{j}\left|\sigma_{n}^{(j)}\right|\left|2^{-k} \sum_{r} \Theta_{k}^{[r]}\left(\mathbf{r}_{k}, \mathbf{h}_{k}(\kappa)\right)^{j}\right| .
$$

Используя теперь (30) и (31), найдем

$$
\left|\sigma_{n+k}-\sigma_{n+k^{\prime}}\right| \leq 2 M e^{G / 2}\left(e^{\kappa^{n} G / 2}-1\right)=M G e^{G / 2} \kappa^{n}+O\left(\kappa^{n}\right) .
$$

Таким образом, взяв $n$ достаточно большим, можно сделать $(20)$ меньше любого $\varepsilon$.

С помошью представленных выше приемов можно также найти

$$
\left|\sigma_{n}-\sigma_{n}^{\#}\right| \leq M e^{G / 2}\left\{\frac{2}{\kappa^{n}} \operatorname{sh}\left(\frac{\kappa^{n}}{2}\right)-1\right\}=\frac{1}{24} M e^{G / 2} \kappa^{2 n}+O\left(\kappa^{2 n}\right),
$$

откуда следует наличие у последовательностей (2), (3) обшего предела при $n \rightarrow \infty$.

Полученные результаты можно сформулировать как 
ПРЕДЛОЖЕНИЕ 2. Если функция $f(x), x \in[0,1]$, дифферениируема любое число раз, причем $(\forall t \in \mathbb{N}):\left|f^{(t)}(1 / 2)\right|<M$, и выполнены условия (7a), последовательности величин (2), (3) сходятся к общему пределу, являющемуся ИМФ 2-го рода.

3.3. Пример расходящегося ИМФ 2-го рода от равномерно непрерывной функции. Покажем, что ИМ $\Phi$ 2-го рода от равномерно непрерывной функции может расходиться. Пусть график функции $f(x)$ имеет вид ломаной с бесконечным числом элементов, последовательно соединяюших точки $L_{*}=\{1,1 / 2\}$ и $L_{q}=$ $\left\{(1 / 2) \kappa^{q},(1 / 2) \kappa^{\rho q}\right\}$ при $q=0,1,2 \ldots ;$ функция $f(x)$, заданная таким образом, равномерно непрерывна при $\rho>0$ и недифференцируема на счетном множестве точек. Для такой функции величина $\sigma_{n}$ может быть найдена в явном виде как сумма вкладов отдельных линейных элементов $L_{q} L_{q-1}, q=1,2, \ldots, n-1$, а также элемента $L_{0} L_{*}$ и точки излома $L_{n}$. Вклад элемента $L_{q} L_{q-1}$ получается, в свою очередь, как сумма по $2^{n-q-1}$ точкам, принадлежашим предфракталу $n$-го поколения и одновременно являющимся вершинами кластера, вершина-предок которого соответствует вектору сигнатуры $\mathbf{s}_{q+1}=(-1, \ldots,-1,+1)$ (способ разбиения графа на кластеры показан на рисунке). Приведем результаты вычислений:

$$
\sigma_{n}=A+B \kappa^{n}+C\left(\beta \kappa^{\rho}\right)^{n},
$$

где

$$
\begin{gathered}
A=\frac{1}{2} \alpha\left(1+\beta \frac{\kappa-2 \kappa^{\rho+1}+\kappa^{\rho}+2 \beta \kappa^{2}\left(\kappa^{\rho}-1\right)}{(1-\kappa)\left(1-\beta \kappa^{\rho}\right)}\right), \\
B=\frac{\alpha \beta(\alpha-\beta)\left(\kappa^{\rho}-1\right)}{2(1-\kappa)\left(1-\beta \kappa^{\rho-1}\right)}, \quad C=\frac{\beta\left(\kappa^{\rho}-1\right)\left(\kappa^{\rho}-\kappa\right)\left(\beta \kappa^{\rho}-2 \alpha \kappa\right)}{2 \kappa^{\rho+1}\left(1-\beta \kappa^{\rho}\right)\left(1-\beta \kappa^{\rho-1}\right)}, \\
\alpha=(1 / 2) \psi(+1), \quad \beta=1-\alpha .
\end{gathered}
$$

Выражение (35) расходится, если $|\beta| \kappa^{\rho}>1$. Поскольку $\kappa$ может быть сколь угодно близким к единице, условие сходимости принимает вид $|\beta|<1$ (в частности, для его выполнения достаточно выполнения условия (7б)).

\section{4. СТРУКТУРА НОСИТЕЛЯ ПРИ РАЗЛИЧНЫХ КОЭФФИЦИЕНТАХ СЖАТИЯ}

Если $\kappa<1 / 2$, величина $\sigma_{n}^{\#}$ является результатом интегрирования по системе непересекаюшихся отрезков $\mathcal{L}_{n}=\cup_{s} l_{n}^{[s]} \subset[0,1]$, где $l_{n}^{[s]}=\left[\lambda_{n}^{[s]}-(1 / 2) \kappa^{n}, \lambda_{n}^{[s]}+(1 / 2) \kappa^{n}\right]$. Множество $\mathcal{L}_{n}$ получается из единичного отрезка путем $n$-кратного повторения стандартной канторовской процедуры: исключается средняя часть длиной $1-2 \kappa$, затем в той же пропорции исключаются средние части получившихся отрезков и т.д. Следуюший шаг - построение меры - состоит в приписывании отрезкам $l_{n}^{[s]}$ весовых множителей $\Theta_{n}^{[s]}$, удовлетворяюших условию (6) и учитываемых при суммировании в (3).

Удобное и наглядное представление о том, что предфрактальный носитель меры $\mathcal{L}_{n}$ и предельное множество $\mathcal{L}=\lim _{n \rightarrow \infty} \mathcal{L}_{n}$ вложены в [0,1], теряется при $1 / 2<\kappa<1$, т.к. в этом случае отрезки $l_{n}^{[s]}$ пересекаются. Эту трудность можно устранить, заменяя отрезки прямоугольниками и "разнося" их вдоль второй координаты. Определим 
двумерньй ИМФ от $f(x, y), x \in[0,1], y \in[0,1]$ :

$$
\iint_{\mathcal{D}} f(x, y) d \mu\left(x, y \mid \kappa_{x}, \kappa_{y}, \Theta\right)=\lim _{n \rightarrow \infty} 2^{-n} \sum_{s} \Theta_{n}^{[s]}\left\langle f\left(\lambda_{x n}^{[s]}, \lambda_{y n}^{[s]}\right)\right\rangle_{\kappa_{x}^{n}, \kappa_{y}^{n}} .
$$

Будем считать, что $\lambda_{\alpha n}^{[s]}$ определяется выражением, получающимся из (4) путем замены $\kappa$ на $\kappa_{\alpha}, \alpha=x, y$, и что усреднение в (36) проводится по области $d_{n}^{[s]}=l_{x n}^{[s]} \otimes l_{y n}^{[s]}, l_{\alpha n}^{[s]}=$ $\left[\lambda_{\alpha n}^{[s]}-(1 / 2) \kappa_{\alpha}^{n}, \lambda_{\alpha n}^{[s]}+(1 / 2) \kappa_{\alpha}^{n}\right]$. Прямоугольные ячейки $d_{n}^{[s]}$ не перекрываются, если хотя бы один из коэффициентов сжатия $\kappa_{\alpha}, \alpha=x, y$, не превышает $1 / 2$. Это условие выполняется, в частности, при $\kappa_{x}=\kappa, 0<\kappa<1$, и $\kappa_{y}=1 / 2$, когда

$$
\iint_{\mathcal{D}} f(x) d \mu(x, y \mid \kappa, 1 / 2, \Theta)=\int_{\mathcal{L}} f(x) d \mu(x \mid \kappa, \Theta) .
$$

Если $\kappa_{\alpha} \leq 1 / 2$ для $\alpha=x$ (для $\alpha=y$ ), проекция $\mathcal{D}=\lim _{n \rightarrow \infty} \cup_{s} d_{n}^{[s]}$ на ось $x$ (ось $y$ ) есть обычное канторовское множество, имеющее размерность подобия $D_{\alpha}=$ $-\left(\log _{2} \kappa_{\alpha}\right)^{-1}$. Размерность всего множества $\mathcal{D}$ можно определить как критическую размерность $d$-меры [9]:

$$
\mathcal{M}_{d}=N(S) S^{d / 2} \stackrel{s \rightarrow 0}{\longrightarrow} \begin{cases}0, & d>D \\ \infty, & d<D\end{cases}
$$

полагая, что $S$ - плошадь ячейки, а $N(S)$ - число таких ячеек $(S \rightarrow 0$ эквивалентно $n \rightarrow \infty)$. При тех значениях параметров, при которых справедливо $(37), D=2 /(1-$ $\left.\log _{2} \kappa\right)$. Заметим, что если к тому же $\kappa=1 / 2$, то $D=1$; в этом случае $\mathcal{D}$ - диагональ единичного квадрата, а (36) - определенный интеграл на этой диагонали.

\section{5. ЗАКЛЮЧЕНИЕ}

Для модельных систем, рассмотренных в разделе 2, ИМФ является формой представления стационарного решения. Здесь мультифракталы, ассоциированные с ИМФ, непосредственно не связаны с модельными предположениями о структуре вешества или фазового портрета. Другой важной областью использования ИМ $\Phi$, отмеченной во введении, может быть представление средних по “физическим" фракталам в модельном приближении. В этом случае согласно $[8,9]$ множители $\Theta_{n}^{[s]}$ приобретают смысл значений концентрации физической величины. Тип распределенной величины: неотрицательная вешественная (пример: масса), вешественная произвольного знака (пример: заряд), комплексная (пример: намагниченность двумерного магнетика) - определяет тип возникающего ИМ $\Phi$; в первом из упомянутых выше случаев мы будем иметь ИМ $\Phi$ 1-го рода, в двух последуюших - 2-го рода. Условия сходимости, найденные в разделе 3 (и, в особенности, тот факт, что ИМФ от негладкой непрерывной функции может не существовать), заставляют предположить, что моделирование самоподобного "физического" распределения канторовским мультифракталом не всегда корректно.

Работа поддержана Российским фондом фундаментальных исследований (грант № 93-02-2011). 


\section{Список литературы}

[1] Nakayama T., Yakubo K., Orbach R.L. // Rev. Mod. Phys. 1994. V. 66. №2. P. 381.

[2] Олемской А.И., Флатm А.Я. // УФН. 1993. Т. 163. №12. С. 1.

[3] Mandelbrot B.B. Fractals and turbulence: attractor and dispersion / Lecture Notes in Mathematics. V. 615. P. 83. Springer-Verlag, 1977.

[4] Mandelbrot B.B. The fractal geometry of nature. N.Y.: Freeman, 1982.

[5] Grassberger P., Procaccia I. // Physica. 1983. V. 9D. P. 189.

[6] Hentschel H.G.E., Procaccia I. // Physica. 1983. V. 8D. P. 435.

[7] Farmer J.D., Ott E., Jorke J.A.// Physica. 1983. V. 7D. P. 153.

[8] Halsey T.C., Jensen M.H., Kadanoff L.P., Procaccia I., Shraiman B.I. // Phys. Rev. 1986. V. 33A. P. 1141.

[9] Федер Е. Фракталы. М.: Мир, 1991.

[10] Kubo R. // J. Phys. Soc. Japan. 1954. V. 9. P. 935.

[11] Anderson P.W. // J. Phys. Soc. Japan. 1954. V. 9. P. 316.

[12] Zverev V.V., Rubinstein B.Ya. // J. Stat. Phys. 1991. V. 63. P. 221.

[13] Ikeda K. // Opt. Comm. 1979. V. 30. P. 257.

[14] Ikeda K., Daido H., Akimoto O. // Phys. Rev. Lett. 1980. V. 45. P. 709.

Институт физики металлов

Уральского отделения

Поступила в редакцию

Российской академии наук

\section{V. Zverev}

\section{ON CONDITIONS OF EXISTENCE OF FRACTAL DOMAIN INTEGRALS}

It is shown that noise transformation processes in some systems with delay may be described in terms of two sorts of the fractal domain integrals. The conditions of convergence of these integrals are analyzed. The structure of multifractals associated with the integrals under the different contraction coefficients is considered. 